\title{
Glucose increases hepatic lipase expression in HepG2 liver cells through upregulation of upstream stimulatory factors 1 and 2
}

\author{
D. van Deursen • H. Jansen • A. J. M. Verhoeven
}

Received: 20 June 2008 / Accepted: 17 July 2008 / Published online: 30 August 2008

(C) The Author(s) 2008. This article is published with open access at Springerlink.com

\begin{abstract}
Aims/hypothesis Elevated hepatic lipase (HL, also known as $L I P C$ ) expression is a key factor in the development of the atherogenic lipid profile in type 2 diabetes and insulin resistance. Recently, genetic screens revealed a possible association of type 2 diabetes and familial combined hyperlipidaemia with the USF1 gene. Therefore, we investigated the role of upstream stimulatory factors (USFs) in the regulation of HL.

Methods Levels of USF1, USF2 and HL were measured in HepG2 cells cultured in normal- or high-glucose medium (4.5 and $22.5 \mathrm{mmol} / \mathrm{l}$, respectively) and in livers of streptozotocin-treated rats.

Results Nuclear extracts of cells cultured in high glucose contained 2.5 \pm 0.5 -fold more USF1 and 1.4 \pm 0.2 -fold more USF2 protein than cells cultured in normal glucose (mean \pm $\mathrm{SD}, n=3)$. This coincided with higher DNA binding of nuclear proteins to the USF consensus DNA binding site. Secretion of HL (2.9 \pm 0.5 -fold), abundance of $H L$ mRNA $(1.5 \pm 0.2$-fold $)$ and $H L(-685 /+13)$ promoter activity $(1.8 \pm$ 0.3 -fold) increased in parallel. In chromatin immunoprecipitation assays, the proximal $H L$ promoter region was immunoprecipitated with anti-USF1 and anti-USF2 anti-
\end{abstract}

D. van Deursen · H. Jansen · A. J. M. Verhoeven $(\bowtie)$

Department of Biochemistry,

Cardiovascular Research School COEUR,

Erasmus MC, P. O. Box 2040, NL-3000 CA Rotterdam,

The Netherlands

e-mail: a.verhoeven@erasmusmc.nl

H. Jansen

Department of Clinical Chemistry,

Cardiovascular Research School COEUR,

Erasmus MC,

Rotterdam, the Netherlands bodies. Co-transfection with USF1 or USF2 cDNA stimulated $H L$ promoter activity 6 - to 16 -fold. USF and glucose responsiveness were significantly reduced by removal of the $-310 \mathrm{E}-$ box from the $H L$ promoter. Silencing of the USF1 gene by RNA interference reduced glucose responsiveness of the $H L(-685 /+13)$ promoter region by $50 \%$. The hyperglycaemia in streptozotocin-treated rats was associated with similar increases in USF abundance in rat liver nuclei, but not with increased binding of USF to the rat $\mathrm{Hl}$ promoter region.

Conclusions/interpretation Glucose increases HL expression in HepG2 cells via elevation of USF1 and USF2. This mechanism may contribute to the development of the dyslipidaemia that is typical of type 2 diabetes.

Keywords Glucose · Hepatic lipase · Human hepatoma cells $\cdot$ Metabolic syndrome $\cdot$ Transcription $\cdot$ Upstream stimulatory factor

\begin{tabular}{|c|c|}
\hline \multicolumn{2}{|c|}{ Abbreviations } \\
\hline AdML & adenovirus major late promoter \\
\hline Apo & apolipoprotein \\
\hline ChIP & chromatin immunoprecipitation \\
\hline ChREBP & $\begin{array}{l}\text { carbohydrate responsive element binding } \\
\text { protein }\end{array}$ \\
\hline FCHL & familial combined hyperlipidaemia \\
\hline HL & hepatic lipase \\
\hline pUSF & upstream stimulatory factor expression plasmid \\
\hline qPCR & quantitative real-time PCR \\
\hline shRNA & short-hairpin RNA \\
\hline shUSF & $\begin{array}{l}\text { plasmid expressing short-hairpin RNA against } \\
\text { upstream stimulatory factor }\end{array}$ \\
\hline SREBP & sterol regulatory element-binding protein \\
\hline USF & upstream stimulatory factor \\
\hline
\end{tabular}




\section{Introduction}

Morbidity and mortality rates in patients with type 2 diabetes are largely dominated by the occurrence of atherosclerotic cardiovascular disease [1]. The insulin-resistant state that precedes the development of type 2 diabetes is also associated with increased risk of cardiovascular disease [2-4]. The dyslipidaemia that typically occurs in insulinresistant states such as central obesity, metabolic syndrome, type 2 diabetes and familial combined hyperlipidaemia (FCHL) $[5,6]$ is an important contributor to the development of coronary artery disease and consists of hypertriacylglycerolaemia, low HDL-cholesterol and a preponderance of small, dense LDL particles $[3,7]$. Hepatic lipase (HL) plays a key role in lipoprotein metabolism and in the remodelling of HDL and LDL [8]. HL is a lipolytic enzyme that mediates the formation of small dense LDL and the reduction of HDLcholesterol levels $[8,9]$. Elevated HL levels are associated with dyslipidaemia in metabolic syndrome and type 2 diabetes [9, 10], and the $H L$ gene (also known as LIPC) is associated with the lipoprotein abnormalities in FCHL [11].

$H L$ expression is increased in type 2 diabetes $[12,13]$. Although insulin-resistant states are commonly associated with hyperinsulinism, a direct stimulating effect of insulin on $H L$ expression has not been unequivocally established [10]. Instead, acute hyperinsulinaemia actually reduces $H L$ expression [14]. HL activity correlates with parameters of insulin resistance in non-diabetic men $[15,16]$ and in FCHL [16]. In an animal model, HL levels were increased upon induction of insulin resistance, which could be partially reversed by treatment with an insulin sensitiser [17]. It is likely therefore that some aspect of insulin resistance induces the increase in $H L$ expression. HL activity is strongly increased with omental fat mass, a parameter of visceral obesity with increased risk of developing type 2 diabetes [18]. This suggests that $H L$ expression may increase with fatty acid supply to the liver. Indeed, in vitro $H L$ expression is increased by fatty acids [19, 20]. Recently, transcription of the $H L$ gene in HepG2 cells was shown to be increased by glucose [21]. Hence, HL levels may be elevated in insulin-resistant states as a consequence of hyperglycaemia and increased fatty acid delivery to the liver.

We recently found that transcription of the $H L$ gene in HepG2 cells was stimulated by the upstream stimulatory factor (USF) 1 [20, 22], a transcription factor that controls expression of several genes involved in glucose and lipid homeostasis [23]. USF1 binds as a homodimer or as a heterodimer with highly homologous USF2, to E-box motifs in gene regulatory sequences. In the liver, expression of the genes coding for glucokinase, fatty acid synthase, apolipoprotein (Apo)A-II, ApoA-V, ApoC-III and ApoE is upregulated by USF [24-29]. In liver as well as in other tissues, USFs play an important role in the regulation of genes by insulin [24-26] or glucose [30-34]. Interestingly, the USF1 gene on chromosome 1q21 has been linked with type 2 diabetes [35], FCHL [36, 37] and both cardiovascular disease and all-cause mortality among women [38]. Allelic variants of USF1 may confer susceptibility to core features of the metabolic syndrome, such as glucose intolerance and dyslipidaemia [36, 39, 40].

USF1 and USF2 are ubiquitously expressed. It is unclear how they convey glucose or insulin responsiveness to susceptible target genes. In USF1 a number of polymorphisms have been reported $[36,38,40]$, some of which are associated with unfavourable results in oral glucose and fat tolerance tests [41], increased adipocyte lipolysis [42] and decreased expression of USF target genes in fat biopsies [40]. In nonhepatic cells, glucose has been shown to increase nuclear expression of either USF1 or USF2 [31-34]. We hypothesised therefore that expression of USF1 or USF2 itself is subject to regulation by glucose. In this study we tested: (1) whether glucose affects nuclear expression of USF proteins in hepatoma cells; and (2) whether this could explain the upregulation of $H L$ expression seen in the high-glucose states.

\section{Methods}

Cell culture and hepatic lipase secretion $\mathrm{HepG} 2$ cells were cultured in DMEM $/ 10 \%$ FCS (vol./vol.) [43], containing either high or normal glucose (all from Gibco BRL, Breda, the Netherlands). Glucose was determined using a sensor (AccuTrend; Roche, Almere, the Netherlands) and was 22.5 and $4.5 \mathrm{mmol} / 1$ in fresh high and normal glucose medium, respectively. Glucose in cell-conditioned media was measured daily. The medium was refreshed every second day or daily if medium glucose had dropped below $1 \mathrm{mmol} / \mathrm{l}$, which occurred occasionally and only when cell cultures were near-confluent. The cultures were split every 5 days. To avoid complications due to acute osmotic effects, cells were maintained at either high or low glucose medium for at least four passages before the start of the experiments.

Hepatic lipase secretion was determined as described [43]. HL activity was expressed as nmol of NEFA released per min from triolein.

RNA analysis Total RNA was isolated from a confluent T25 flask of HepG2 cells using the TRIzol reagent (Invitrogen, Leek, the Netherlands). The amount of $H L$ mRNA was quantified by reverse-transcription followed by quantitative real-time PCR (qPCR) as described previously [43]. $H L$ mRNA levels were normalised to acidic ribosomal phosphoprotein PO (36B4) mRNA.

Determination of USF1 and USF2 expression Nuclear extracts were prepared from the cells of one confluent 
T25 culture flask and the proteins were analysed by immunoblotting as described previously [43]. USF1 and USF2 protein were detected by a 1:500 dilution of rabbit anti-human USF1 and anti-human USF2 (both C20; SantaCruz Biotechnology, Santa Cruz, CA, USA), respectively. B23 protein was detected by 1:10,000 dilution of mouse monoclonal anti-B23. Bound rabbit and mouse antibodies were detected by a 1:5,000 dilution of the respective horseradish peroxidase-coupled secondary antibodies.

Human USF1 and USF2 proteins were synthesised in vitro using the TnT reticulocyte lysate system (Promega, Leiden, the Netherlands) according to the manufacturer's instructions, with USF1 cDNA and USF2 cDNA in pcDNA3 (pUSF1 and pUSF2) as expression vectors, respectively. pUSF1 was obtained from B. Staels (Pasteur Institute, Lille, France). The entire coding sequence of USF 2 cDNA was generated by RT-PCR on total HepG2 RNA using 5'-gcgaattCCATGGA CATGCTGGAC-3' as forward primer and 5'-gctctaga GCGTGGTGGTGGCGG-3' as reverse primer. The extra EcoRI and $\mathrm{XbaI}$ restriction sites (sequences underlined) were used for cloning into pcDNA3. The insert was verified by DNA sequencing (BaseClear, Leiden, the Netherlands).

Gelshift assays Gelshift assays using ${ }^{32} \mathrm{P}$-labelled probes were performed as described previously [22]. Assays using biotinylated probes were performed with a kit (LightShift Chemiluminescent EMSA Kit; Pierce, Rockford, IL, USA). In supershift assays, the nuclear extract protein was preincubated for $30 \mathrm{~min}$ on ice with $1 \mu \mathrm{l}$ of anti-USF1 (C-20), anti-USF2 (C-20) or anti-sterol regulatory element-binding protein (SREBP)2 (N-19; Santa-Cruz).

Measurement of HL promoter activity Transcriptional activity of the human $H L(-685 /+13)$ promoter fragment was determined by transient transfection of the HepG2 cells with the $H L-685$ luciferase vector as described previously [20], except that the medium was also refreshed at $24 \mathrm{~h}$ post transfection. Co-transfection with pRL-TK (Promega) was used as internal control and luciferase activity data were normalised on the basis of Renilla activity. Each transfection assay was performed in quadruplicate.

From the $H L(-685 /+13)$ promoter construct in pGL3, the 5'-deleted $H L-305$ promoter construct was prepared by PCR using appropriate oligonucleotide primers. In the $H L-$ 685 construct, the -310 E-box CACGTG was scrambled by PCR-directed mutagenesis into GCTAGC (Em). Similarly, the -514 E-box CACGGG $(-514 \mathrm{C})$ was changed into CATGGG $(-514 T)$. Mutations were verified by automated sequencing (BaseClear).

RNA interference analysis RNA silencing of USF1 and USF2 was performed by transient transfection with pSilencer 3.1-H1 hygro expression vector (Ambion, Austin
TX, USA), which generates short-hairpin RNA (shRNA) molecules. The sequences of the shRNA molecules directed against USF1 and USF2 partially overlapped with the targeting sites used by McMurray and McCance [44]. The sequences of the shRNA molecules were: for USF1 5'GGTGGGATTCTATCCAAAGCTTCAAGAGAGCTTTG GATAGAATCCCACCTTTTTT-3'; and for USF2 5'-GGA GATACTACGGCTGTGTCCAAGCTTGGACAC AGCCGTAGTATCTCCTTTTT-3'. As controls, parallel transfections were performed with empty pSilencer vector or with $\mathrm{pSilencer}$ constructs targeted against two non-related genes $(A Q P 3$ and $A Q P 7)$.

Chromatin immunoprecipitation assays Chromatin immunoprecipitation (ChIP) assays were performed as described previously [43] using anti-USF1 or anti-USF2 antibodies (Santa Cruz). The immunoprecipitated DNA was subjected to qPCR; amplified regions of the human $H L$ gene were 480/-352 (primers 5'-CACAAGCATCACCAATTTCAC-3' and 5'-GCTGGCTCAGGAAAGTGG-3') and -6071/-5986 (primers 5'-CTTGGGATTTGCTTGCTTTATC-3' and 5'ATTTGATGACCTGAGAATGACC-3'). Alternatively, the PCR products were resolved by $3 \%(\mathrm{wt} / \mathrm{wt})$ agarose gel electrophoresis and visualised by ethidium bromide staining.

Livers from diabetic rats Female Wistar rats weighing 250 to $350 \mathrm{~g}$ were housed in a reversed light-cycle room and had free access to water and standard rodent chow. Animal experiments were carried out according to the regulations of the local Animal Care and Use Committee. Animals were made diabetic by a single intraperitoneal injection of $55 \mathrm{mg} / \mathrm{kg}$ of streptozotocin (Sigma, St Louis, IL, USA) in $100 \mathrm{mmol} / \mathrm{l}$ sodium citrate ( $\mathrm{pH} 4.5$ ) [45]. Control animals were injected with vehicle only. At 7 weeks, rats were killed by an isoflurane overdose. Glucose was determined in full blood samples. Liver portions were quickly removed into liquid nitrogen and stored at $-80^{\circ} \mathrm{C}$ until further analysis. For immunoblotting assays, nuclear extracts were prepared from frozen $100 \mathrm{mg}$ liver aliquots. Of each extract, $50 \mu \mathrm{g}$ protein was tested for USF1 and USF2 as described above and $1 \mu \mathrm{g}$ for histone $\mathrm{H} 3$ using rabbit antiH3 antibody (1:2500; Abcam, Cambridge, UK).

For ChIP assays, $100 \mathrm{mg}$ liver aliquots were powdered in liquid nitrogen and immediately thawed in $1 \mathrm{ml}$ of $1 \%$ (wt/wt) formaldehyde in PBS. After 10 min incubation at $37^{\circ} \mathrm{C}$, the homogenate was diluted once in $2 \%(\mathrm{wt} / \mathrm{wt}) \mathrm{SDS}$, $20 \mathrm{mmol} / \mathrm{l}$ EDTA, $100 \mathrm{mmol} / \mathrm{l}$ Tris-HCl (pH 8.0). ChIP assays were performed as described for HepG2 cells [43]. Amplified regions of the rat $\mathrm{Hl}$ gene were $-392 /-282$ (primers 5'-GGGGCTTTTACCTCTCTTTGGG-3' and 5'TGACCTCTGTATTGTTGCCTGTG-3') and -7328/-7214 (primers 5'-CACAGCAACACGAGCCTCAG-3' and 5'TGCAGTGTAGAATTTGTGGCATACC-3'). For determi- 
nation of HL activity, frozen $100 \mathrm{mg}$ liver aliquots were homogenised in $2 \mathrm{ml}$ heparin containing PBS on ice using a homogeniser (UltraTurrax T25; IKA, Staufen, Germany). HL activity was determined in the homogenate [43] and was completely inhibited by preincubation with excess antirat $\mathrm{HL}$ antiserum.

Statistics All data are expressed as means \pm SD. Statistical analyses were performed using Student's $t$ test. Statistical significance was defined as $p<0.05$.

\section{Results}

Glucose increases both HL expression and nuclear accumulation of USF1 and USF2 HepG2 cells that had been cultured to confluence at $22.5 \mathrm{mmol} / 1$ glucose were incubated for an additional $8 \mathrm{~h}$ in medium containing normal $(4.5 \mathrm{mmol} / \mathrm{l})$, intermediate $(9 \mathrm{mmol} / \mathrm{l})$ or high (22.5 mmol/l) glucose. Secretion of HL increased with increasing glucose concentration (Fig. 1a). In the highglucose medium, HL secretion was $2.9 \pm 0.5$-fold higher than in the normal-glucose medium. After $8 \mathrm{~h}$ of incubation under these conditions (confluent wells and reduced medium volume), glucose was completely depleted from the normal-glucose medium. In the high-glucose medium, glucose was reduced to $13.0 \pm 1.9 \mathrm{mmol} / \mathrm{l}(n=3)$. In medium with intermediate glucose concentration, extracellular glucose decreased from 9 to $1.6 \pm 0.3 \mathrm{mmol} / 1 \quad(n=3)$. HL secretion was also intermediate between the normal- and high-glucose cells. In further experiments, cells were incubated with higher volumes of medium per well, with the medium being refreshed daily to keep extracellular glucose above $1 \mathrm{mmol} / \mathrm{l}$ throughout the incubation. Under these conditions, the abundance of $H L$ mRNA, as determined by qPCR, in high-glucose cells was $1.5 \pm 0.2$-fold higher than in parallel normal-glucose cells $(p=0.028, n=$ 3 ). To test the effect of glucose on $H L$ transcription, cells were transiently transfected with the $H L-685$ luciferase promoter-reporter construct. Luciferase activity in the highglucose cells was $1.8 \pm 0.3$-fold higher than in the normalglucose cells $(p=0.036 ; n=3)$. This suggests that glucose dose-dependently increases HL secretion, largely by affecting transcription of the $H L$ gene.

The amount of USF1 protein in nuclear extracts prepared from high-glucose cells was $2.5 \pm 0.5$-fold higher than that in the normal-glucose cells medium $(n=3, p=0.042)$ (Fig. 1b). USF2 was slightly, but significantly, higher in the highglucose cells $(1.4 \pm 0.2$-fold, $n=3, p=0.010)$. In contrast, the amount of B23 protein was similar in both nuclear extracts.

Glucose increases DNA binding activity of USF In gelshift assays with HepG2 nuclear extracts and adenovirus major late promoter (AdML), the USF consensus-binding site, a similar shift was produced with high- and normal-glucose cells (Fig. 2a). With the high-glucose cells, markedly more probe was shifted than with the normal-glucose cells. The shifted band co-migrated with in-vitro synthesised USF1 and USF2 proteins run in parallel. In supershift assays, the band was partially shifted to higher positions in the gel after

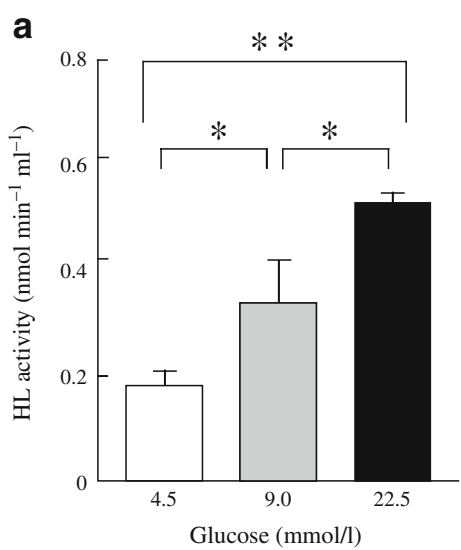

b

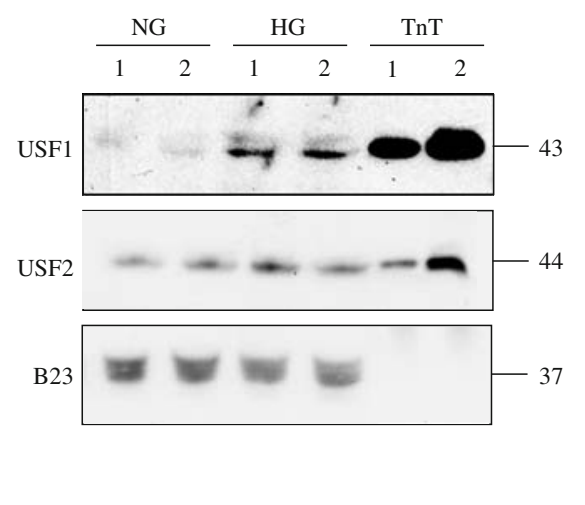

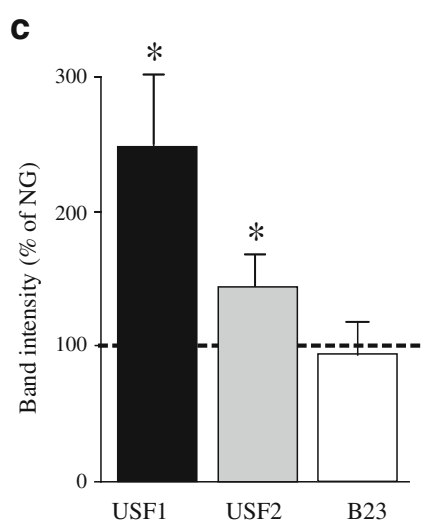

Fig. 1 High glucose increases HL secretion and nuclear accumulation of USF proteins. a HepG2 cells were incubated for $8 \mathrm{~h}$ in medium containing heparin and the indicated amounts of glucose. At the end of incubation, HL activity was determined in the cell-free media. Data are mean \pm SD for three to five independent experiments. b HepG2 cells were cultured with 4.5 (normal glucose [NG]) or $22.5 \mathrm{mmol} / \mathrm{l}$ (high glucose [HG]) glucose. At confluence, nuclear extracts were prepared and the presence of USF1 and USF2 protein was detected by immunoblotting (in duplicate). B23 protein served as internal control. TnT 1 and 2 are 0.5 and $1.0 \mu \mathrm{l}$ respectively of in vitro transcribed and translated USF, and served as positive controls. Molecular masses are in $\mathrm{kDa}$. $\mathbf{c}$ The data (b) were quantified by densitometry, the two parallels averaged and data expressed as percentage of NG controls. Data are mean \pm SD for three to four independent experiments. ${ }^{*} p<0.05,{ }^{* *} p<0.01$ 


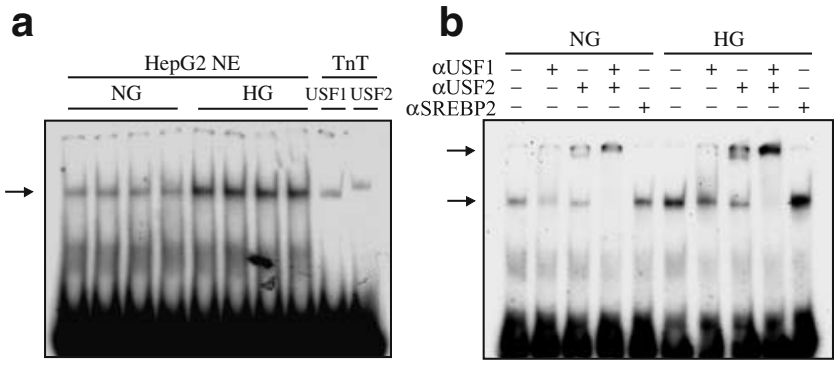

Fig. 2 Glucose increases E-box DNA binding activity in HepG2 nuclei. a Gelshift and (b) supershift assays were performed using ${ }^{32} \mathrm{P}-$ labelled AdML oligonucleotides containing consensus USF binding sites and nuclear extracts $(2.5 \mu \mathrm{g}$ protein $)$ from HepG2 cells that had grown to confluence in normal glucose (NG) or high glucose (HG). a Nuclear extracts (NE) from four different incubations were run in parallel and with TnT reticulolysate extracts that had been incubated with pUSF1 or pUSF2. b The nuclear extracts were pre-incubated with the antibody indicated prior to the probe. Arrows indicate the positions of the shifted and supershifted bands

preincubation of the nuclear extracts with antibodies against USF1 or USF2 (Fig. 2b), but not with antibodies against SREBP2. A complete supershift was induced by preincubation with anti-USF1 and anti-USF2 combined. These data indicate that glucose increases the amount and DNA binding activity of USF proteins in HepG2 cell nuclei and suggest that the USF proteins induced by glucose are active in DNA binding.

Glucose increases USF1 and USF2 binding to the HL promoter in chromatin The proximal $H L$ promoter region co-immunoprecipitated with both anti-USF1 and anti-USF2 (Fig. 3a). Significantly more PCR product was generated with DNA immunoprecipitated from high-glucose than from normal-glucose cells using primers specific for the proximal $H L$ promoter region (-480/-352) (Fig. 3b). In contrast, the amount of PCR product generated with distal $H L$ gene-specific primers $(-6071 /-5965)$ was negligible and not affected by glucose conditioning. Hence, both USF1 and USF2 are bound to the proximal $H L$ promoter region in the HepG2 nuclei in situ and binding is higher in high-glucose than in normal-glucose cells.

Glucose-induced increase of HL promoter activity is reduced by removal of potential USF binding sites Cotransfection of $H L-685$ with 10 and $24 \mathrm{ng}$ of pUSF1 increased luciferase activity approximately 6 - and 16-fold, respectively (Fig. 4b,c). In this $H L$ promoter fragment, E-boxes that potentially bind USF are present around positions -514 and -310 . We were not able to show binding of endogenous USFs to these sites in gelshift assays with HepG2 nuclear extracts, in agreement with the low binding affinity compared with the AdML consensus site [22]. However, purified USFs have been shown to bind

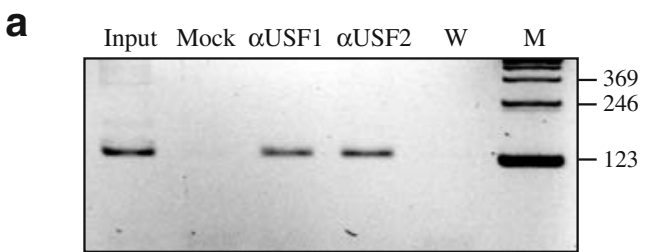

b

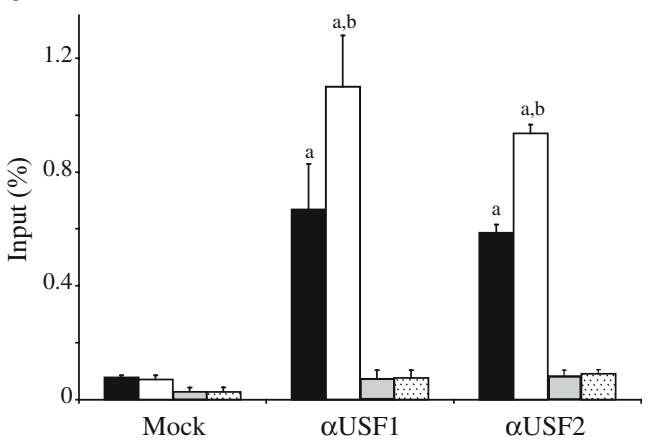

Fig. 3 Chromatin immunoprecipitation assays with HepG2 cells. Chromatin was immunoprecipitated from HepG2 cells with anti-USF1 or anti-USF2 and the DNA was analysed by PCR using $H L$-specific primers. a Part of the proximal $H L$ promoter region $(-480 /-352)$ was amplified $\left(30\right.$ cycles at $30 \mathrm{~s} 95^{\circ} \mathrm{C}, 30 \mathrm{~s} 55^{\circ} \mathrm{C}$ and $\left.45 \mathrm{~s} 72^{\circ} \mathrm{C}\right)$ using $4 \mu \mathrm{l}$ immunoprecipitate, and PCR products were analysed by agarose gel electrophoresis and ethidium bromide staining. As positive and negative controls, $1 \%$ input DNA and no-antibody immunoprecipitation (Mock) were included, respectively. W, PCR water control; M, the 123 bp DNA ladder from Roche. b DNA immunoprecipitated with the indicated antibody from normal-glucose (black and grey bars) and high-glucose (white and stippled bars) cells was analysed by qPCR, amplifying either the $-480 /-352$ (black and white bars) or -6071 / -5965 (grey and stippled bars) regions of the $H L$ gene. Data are mean \pm SD of three independent experiments. ${ }^{a, b}$ Statistically significant difference from mock and normal glucose, respectively

to the -514 region of the HL promoter [22], and Fig. $4 \mathrm{~d}$ demonstrates binding of USF1 and USF2 to the -310 region in nuclear extracts of HepG2 cells transiently transfected with the respective expression plasmids. Mutation of the -514 E-box from CACGGG $(-514 \mathrm{C})$ into the allelic variant CATGGG (-514T) reduced responsiveness of the $H L-685$ construct to USF1 and USF2, but this reduction did not reach statistical significance (Fig. 4b). Scrambling of the -310 E-box sequence from CACGTG $(-310 \mathrm{E})$ to GCTAGC $(-310 \mathrm{Em})$ reduced responsiveness to USF 1 and USF 2 to approximately $50 \%$ ( $p=0.006$ and $p=$ 0.001 , respectively; $n=4$ ) (Fig. $4 \mathrm{c}$ ). This suggests that USF1 and USF2 upregulate $H L$ promoter activity in part through binding at the $-310 \mathrm{E}-\mathrm{box}$.

Compared with $H L-685$, the $H L-305$ construct lacks both potential USF binding sites at -514 and -310 . Both reporter constructs were tested in parallel for promoter activity. Whereas in this series of experiments the activity of $H L-685$ was $2.4 \pm 0.6$-fold higher in the high-glucose than in the normal-glucose cells, this value was only $1.6 \pm 0.3$-fold 
a

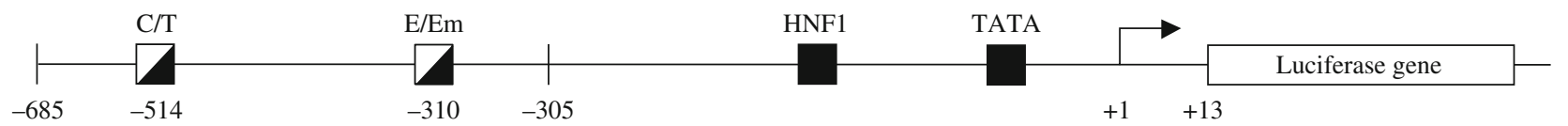

b

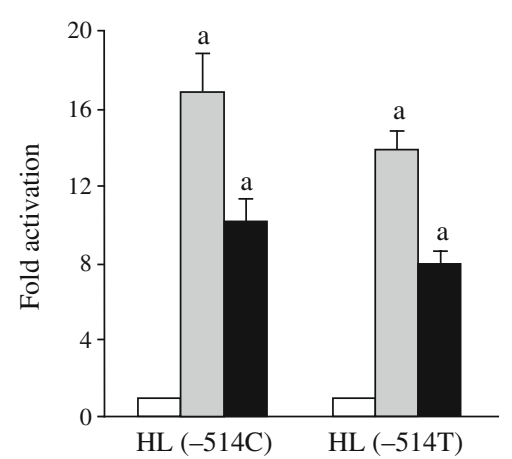

C

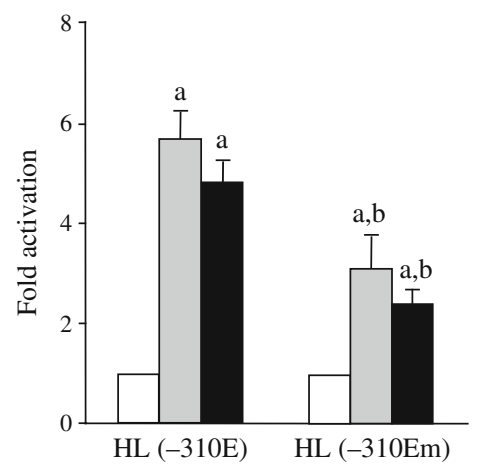

d

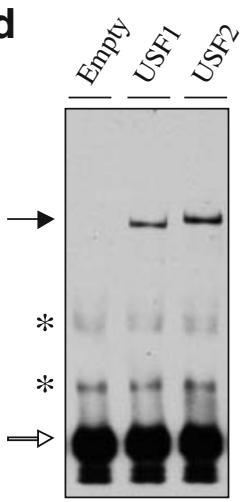

e

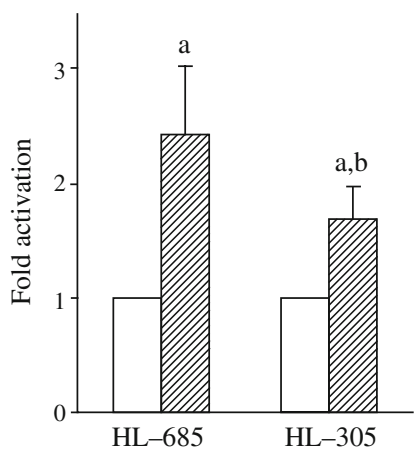

Fig. 4 E-box motifs in the $H L$ promoter fragment are required for full responsiveness to USF1 and glucose. a Schematic representation of the $H L(-685 /+13)$ promoter fragment, indicating the position of two potential USF-binding E-box motifs. The role of these E-boxes was tested either by mutating them $(\mathbf{b}, \mathbf{c})$ or by removing both E-boxes by 5 '-deletion (e). The effect (b, c) of the $-514 \mathrm{C} \rightarrow \mathrm{T}$ mutation or the $-310 \mathrm{E} \rightarrow$ Em mutation was tested on responsiveness to stimulation with USF1 and USF2 in transient co-transfection assays. High-glucose HepG2 cells were co-transfected with $24 \mathrm{ng}$ (b) or $10 \mathrm{ng}$ (c) of pUSF1 (grey bars) and pUSF2 (black bars). Luciferase activities were expressed relative to the activities measured in cells co-transfected with empty pcDNA3 vector (control, white bars). ${ }^{\mathrm{a}, \mathrm{b}}$ Statistically significant difference from control cells and from the wild-type

with the shorter construct ( $p=0.037 ; n=5$ ) (Fig. 4e). Hence, the E-boxes in the $H L$ promoter are also important for full glucose responsiveness.

Glucose-induced increase of HL promoter activity is reduced by silencing of USF1 Transfection of HepG2 cells with pSilencer plasmids that code for shRNA directed against USF1 and USF2 markedly reduced the amount of USF1 and USF2 protein in nuclear extracts, respectively (Fig. 5a). In normal-glucose cells, $H L$ promoter activity was slightly but significantly reduced by co-transfection with short-hairpin USF (shUSF)1 and shUSF2 (Fig. 5b). In the high-glucose cells, the $H L$ promoter activity was reduced to approximately $50 \%$ of parallel controls, with upregulation by high-glucose almost completely prevented by cotransfection with shUSF1. Similar trends were observed with shUSF2, but the effects did not reach statistical significance. shRNA constructs directed against non-related $A Q P 3$ or $A Q P 7$ did not affect $H L$ promoter activity in the high-glucose cells (Fig. 5c).

$\mathrm{Hl}$ expression in livers of streptozotocin-treated rats To test whether chronically elevated glucose affects $U s f$ and $\mathrm{Hl}$ expression in vivo, we used livers of streptozotocin-treated construct, respectively. d A gelshift of biotinylated -300/-320 region of the $H L$ gene and nuclear extracts of HepG2 cells that were transiently transfected with 100 ng of pUSF1 or pUSF 2 or with empty vector. Arrows, position of the shifted bands; *, non-specific bands. e The glucose responsiveness of HL-685 compared with HL-305. HepG2 cells grown in normal (white bars) or high (hatched bars) glucose were transiently transfected with both promoter constructs and the luciferase activity was determined. Activities in the normalglucose cells were set at 1.0. Activities in the high-glucose cells were expressed as fold increase relative to normal-glucose cells. Data are mean $\pm \mathrm{SD}$ of four to five independent experiments. ${ }^{\mathrm{a}, \mathrm{b}}$ Statistically significant difference from normal-glucose cells and from the fulllength construct, respectively

rats. Whole-blood glucose concentration in streptozotocintreated rats was $23.9 \pm 1.2 \mathrm{mmol} / 1$ compared with $5.9 \pm$ $0.5 \mathrm{mmol} / 1$ in control rats $(p<0.05 ; n=3)$. In livers of the treated rats, nuclear expression of USF1 was 2.1 -fold ( $p<$ $0.05 ; n=3)$ higher and that of USF2 was 1.4 -fold $(p<0.05$; $n=3$ ) higher than in parallel controls (Fig. 6a). In ChIP assays, the proximal region of the rat $\mathrm{Hl}$ gene $(-392 /-282)$ was immunoprecipitated with anti-USF1 and anti-USF2, but not a distal region of the $\mathrm{Hl}$ gene (-7329/-7214), indicating that USF proteins are specifically bound to the proximal promoter region of $\mathrm{Hl}$ in rat liver (Fig. 6b). Binding of USF proteins to the upstream region of $\mathrm{Hl}$ was not significantly affected by high glucose levels, as similar amounts of DNA were immunoprecipitated from livers of streptozotocin-treated and control rats. HL activity in liver homogenates amounted to $4.3 \pm 0.4$ and $10.6 \pm 2.8 \mathrm{nmol}$ $\min ^{-1}$ (mg protein $)^{-1}$ in streptozotocin-treated and control rats, respectively $(p<0.05 ; n=3)$.

\section{Discussion}

Our study shows that HepG2 cells contain more USF1 and USF2 protein in their nuclei under high- than under low- 
a

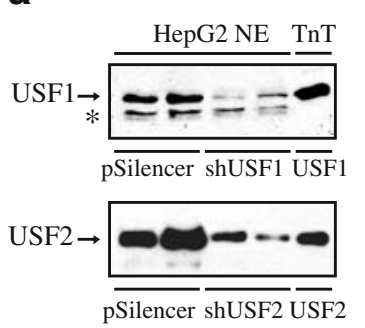

C

b

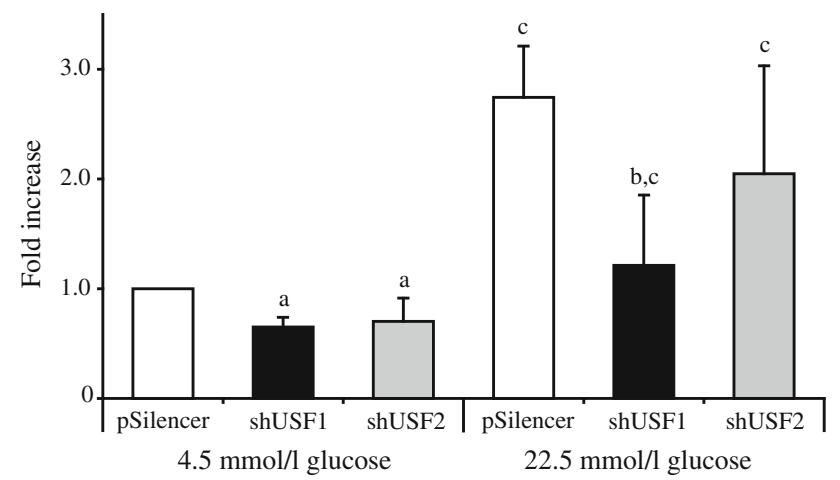

Fig. 5 Silencing USF1 reduces glucose-induced upregulation of $H L$ promoter activity. HepG2 cells were co-transfected with $H L-685$ and $100 \mathrm{ng}$ of the indicated shRNA construct in pSilencer. a Effect of shUSF1 on endogenous USF1 protein and of shUSF2 on endogenous USF2 protein in nuclear extracts as indicated. Nuclear protein $(25 \mu \mathrm{g})$ was run in parallel with in vitro synthesised USF protein (TnT). *, cross-reacting band. b $H L-685$ promoter activity was determined in cells co-transfected with $100 \mathrm{ng}$ shUSF1, shUSF2 or pSilencer. Luciferase data were expressed as fold stimulation relative to the activity with pSilencer measured in normal-glucose cells. Data are mean $\pm \mathrm{SD}$ of five independent experiments. c The effect of the shUSF1 construct was compared with shRNA constructs directed against $A Q P 3$ and $A Q P 7$. Cells were incubated in high-glucose medium. Data are means \pm SD of five parallel incubations. ${ }^{a, b}$ Statistically significant differences of sh vs pSilencer in normal- and highglucose conditions, respectively; 'statistically significant differences between high and normal glucose

glucose conditions. As extracellular glucose concentrations in our experiments frequently reached very low levels, it could be argued that USF proteins disappear from the nuclei in low-glucose conditions. However, we favour the interpretation that high glucose induces the nuclear accumulation of USF proteins, as increased expression of both USF proteins was also observed in livers of hyperglycaemic, streptozotocin-treated rats. In HepG2 cells, this was paralleled by increased binding of USFs to the $H L$ promoter region and a parallel increase in $H L$ expression. The human $H L$ gene is a potential target for USF, as (1) USF proteins were bound to the proximal promoter region in HepG2 cells (Fig. 3), (2) this region contains functionally important Eboxes and (3) $H L$ promoter activity was dose-dependently upregulated by overexpression of USF1 or USF2 (Fig. 4). Silencing of USF1 by RNA interference strongly reduced the glucose-induced upregulation of $H L$ promoter activity a

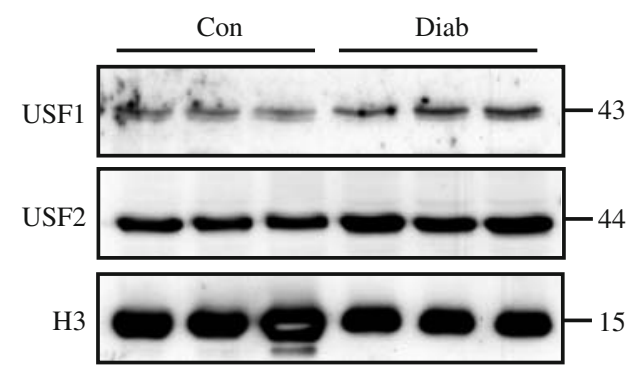

b

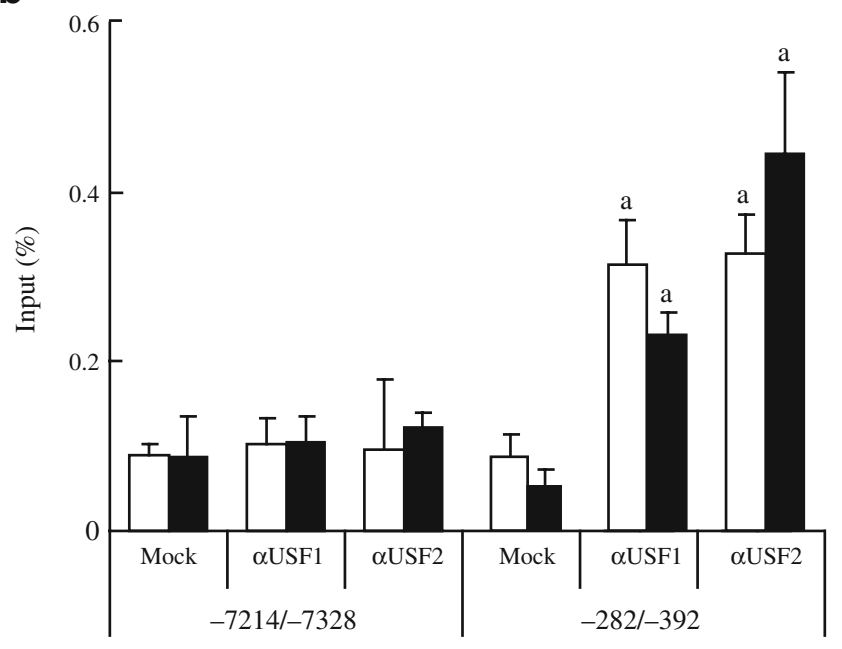

Fig. 6 Effect of chronically elevated blood glucose on USF expression and ChIP in rat liver. a Expression of USF1 and USF2 was determined in liver nuclear extracts of three control rats (Con) and three streptozotocin-treated rats (Diab), using histon $\mathrm{H} 3$ as loading control. The normalised signal densities (arbitrary units) were $19.0 \pm$ 2.8 vs $9.0 \pm 0.9$ for USF1 and $72.7 \pm 11.5$ vs $51.0 \pm 5.7$ for USF2 in treated vs control livers, respectively $(n=3)$. Molecular masses are indicated in $\mathrm{kDa}$. b ChIP assays were performed with chromatin isolated from livers of control (white bars) and streptozotocin-treated (black bars) rats and with anti-USF1, anti-USF2 or no (Mock) antibodies. Immunoprecipitated DNA was analysed by qPCR using rat $\mathrm{Hl}$-specific primers targeted at the indicated regions. Data are mean $\pm \mathrm{SD}$ of three independent experiments. ${ }^{\text {a }}$ Statistically significant difference from mock

(Fig. 5). Therefore, the USF proteins that accumulate in the nuclei of HepG2 cells are important in mediating the glucose-induced increase in $H L$ expression. Glucose responsiveness of the $H L$ promoter was not completely lost upon deletion of the E-boxes. Hence, the glucose-induced upregulation of $H L$ promoter activity may be mediated by USFs via additional, non-E box elements. Co-transfection with pUSF1 and pUSF2 also increased transcription of proximal $H L$ promoter constructs through binding of USFs to the transcription initiation site (D. van Deursen, H. Jansen and A.J.M. Verhoeven, unpublished results). In rat liver chromatin, USF1 and USF2 protein apparently bind to the proximal $\mathrm{Hl}$ promoter region at non-E box elements, as this region of the rat $\mathrm{Hl}$ gene lacks canonical E-boxes. Alternatively, glucose may activate the $H L$ promoter 
through additional mechanisms that are independent of USFs. Recently, carbohydrate responsive element binding protein (ChREBP) was identified as a major glucoseresponsive transcription factor in liver cells; its consensus DNA binding site, however, consists of two E-boxes separated by 5 bp [46]. Using MatInspector (matrix similarity $>0.82$ ) [47], we found such a potential binding site within $2 \mathrm{~kb}$ of the proximal USF1 and USF2 but not of the $H L$ promoter region. Moreover, fatty acids decrease expression of glucose-responsive gene through decrease of ChREBP activity [48], whereas fatty acids increased HL $[19,20]$ and nuclear USF expression (D. van Deursen and A. J. M. Verhoeven, unpublished observations) in HepG2 cells. A major role for ChREBP in mediating glucoseresponsiveness to $H L$ therefore appears unlikely.

In contrast to HepG2 cells, the accumulation of USF1 and USF2 in liver nuclei of streptozotocin-treated rats was not accompanied by increased binding of USFs to the proximal $\mathrm{Hl}$ promoter region. In addition, $\mathrm{Hl}$ expression was reduced in this in vivo model. Apparently, increased abundance of USF proteins is not the only factor that regulates USF binding activity and $H L$ expression. In the in vivo model, liver cells are not only chronically exposed to hyperglycaemia and low insulin, but also to many other variables not mimicked in our in vitro model, e.g. elevated fatty acid levels, glucocorticoids and glucagon. Our preliminary data show that addition of $1 \mathrm{mmol} / \mathrm{l} \mathrm{BSA-}$ bound oleate to the high-glucose medium further increased USF1 expression, $H L$ promoter activity and secretion of $\mathrm{HL}$ in HepG2 cells. In contrast, glucocorticoids and glucagon lower $\mathrm{Hl}$ expression in rat hepatocytes [49, 50], possibly through mechanisms that override the positive effect of increased nuclear levels of USFs. One possibility is that USFs become phosphorylated, thereby affecting their DNA binding activity [23, 26]. Whether increased nuclear expression of USF protein leads to increased USF binding to the proximal $H L$ promoter and subsequently to upregulation of $H L$ expression apparently depends on the species studied or on the prevailing metabolic or hormonal status. Alternatively, the hyperglycaemia-induced, USF-mediated upregulation of $H L$ expression may be restricted to human hepatoma cells.

USF1 and USF2 are ubiquitously expressed. In various non-hepatoma cells, glucose has been shown to increase either nuclear USF1 or USF2 or both [32-34]. In HepG2 cells, glucose increases predominantly nuclear USF1, and silencing of USF1 but not USF2 abolished the increase in HL promoter activity. In mouse liver, glucose responsiveness is mainly signalled through the USF1-USF2 heterodimer, but the homodimers are also transcriptionally active [30]. Hence, upregulation of either USF1 or USF2 may affect expression of target genes. The relative importance of USF1 and USF2 overproduction may depend on which of the two proteins is limiting in the cell type used. Taken together, our data suggest that the nuclear accumulation of USF1 and USF2 signals glucose responsiveness to the $H L$ gene and probably to other USF target genes. The mechanism that leads to accumulation of USF1 and USF2 by glucose is presently unknown. In adipocytes, glucose has to be metabolised in the glycolytic pathway beyond glucose6-phosphate to triose phosphates [31]. Further research is required to show whether this also holds for liver cells.

USF1 and USF2 are involved in the coordinate regulation of glucose and lipid metabolism [23, 40], in which the liver plays a central role. The finding of increased nuclear expression of USF proteins may therefore bear relevance to the causes of insulin resistance and type 2 diabetes. The associated dyslipidaemia (low HDL, high triacylglycerol, increased small dense LDL) may result from the increased transactivation by USFs, not only of $H L$, but also of other HDL- and triacylglycerol-related genes [26-29, 31]. Increased USF1 transactivation of its target genes has been suggested to explain the development of the metabolic syndrome [35, 39], the dyslipidaemia associated with FCHL $[36,40]$ and the development of diabetic complications [32, 34]. Metabolic syndrome, type 2 diabetes and FCHL have all been linked to the USF1 gene [35-37]. A number of risk alleles of USF 1 have been identified, which all represent variants of the non-coding sequence [36, 38, 41]. It is not clear how non-coding polymorphisms in USF 1 may contribute to these metabolic disorders. The polymorphism in intron 7 of USF1 [36] has been shown to affect binding of nuclear proteins to this region [38], suggesting that this polymorphism affects transcriptional or posttranscriptional regulation of USF1 expression. Possibly, these polymorphisms interfere with the glucose-mediated nuclear accumulation of USF1 in the liver. Further studies are required to elucidate the mechanism by which glucose affects USF1 and USF2 expression and how genetic variants of USF1 may alter responsiveness to glucose.

Acknowledgements We thank P. K. Chan (Baylor College of Medicine, Houston, TX, USA) for kindly providing the monoclonal anti-B23 antibody, B. Staels for the USF1 expression vector and B. Tilly and C. Lim (Erasmus MC, Rotterdam, the Netherlands) for the $A Q P 3$ and $A Q P 7$ shRNA constructs. We are grateful to J. M. J. Lamers and $\mathrm{H}$. A. van Heugten for allowing us to use the livers and data of control and streptozotocin-treated rats. This study was supported in part by the Netherlands Heart Foundation (grant number 2001B174).

Duality of interest The authors declare that there is no duality of interest associated with this manuscript.

Open Access This article is distributed under the terms of the Creative Commons Attribution Noncommercial License which permits any noncommercial use, distribution, and reproduction in any medium, provided the original author(s) and source are credited. 


\section{References}

1. Varughese GI, Tomson J, Lip GY (2005) Type 2 diabetes mellitus: a cardiovascular perspective. Int J Clin Pract 59:798-816

2. Sobel BE, Schneider DJ (2005) Cardiovascular complications in diabetes mellitus. Curr Opin Pharmacol 5:143-148

3. Reaven GM (2005) Compensatory hyperinsulinemia and the development of an atherogenic lipoprotein profile: the price paid to maintain glucose homeostasis in insulin-resistant individuals. Endocrinol Metab Clin North Am 34:49-62

4. Nigro J, Osman N, Dart AM, Little PJ (2006) Insulin resistance and atherosclerosis. Endocr Rev 27:242-259

5. Bredie SJ, Tack CJ, Smits P, Stalenhoef AF (1997) Nonobese patients with familial combined hyperlipidemia are insulin resistant compared with their nonaffected relatives. Arterioscler Thromb Vasc Biol 17:1465-1471

6. Brunzell JD, Hokanson JE (1999) Dyslipidemia of central obesity and insulin resistance. Diabetes Care 22(Suppl 3):C10-C13

7. Ginsberg HN (2006) Review: Efficacy and mechanisms of action of statins in the treatment of diabetic dyslipidemia. J Clin Endocrinol Metab 91:383-392

8. Jansen H, Verhoeven AJM, Sijbrands EJG (2002) Hepatic lipase: a pro- or anti-atherogenic protein? J Lipid Res 43:1352-1362

9. Deeb SS, Zambon A, Carr MC, Ayyobi AF, Brunzell JD (2003) Hepatic lipase and dyslipidemia: interactions among genetic variants, obesity, gender, and diet. J Lipid Res 44:1279-1286

10. Rashid S, Watanabe T, Sakaue T, Lewis GF (2003) Mechanisms of HDL lowering in insulin resistant, hypertriglyceridemic states: the combined effect of HDL triglyceride enrichment and elevated hepatic lipase activity. Clin Biochem 36:421-429

11. Allayee H, Dominguez KM, Aouizerat BE et al (2000) Contribution of the hepatic lipase gene to the atherogenic lipoprotein phenotype in familial combined hyperlipidemia. J. Lipid Res 41:245-252

12. Tan KCB, Shiu SWM, Chu BYM (1999) Roles of hepatic lipase and cholesteryl ester transfer protein in determining low density lipoprotein subfraction distribution in Chinese patients with non-insulin-dependent diabetes mellitus. Atherosclerosis $145: 273-278$

13. Berk-Planken II, Hoogerbrugge N, Stolk RP, Bootsma AH, Jansen $\mathrm{H}$ (2003) Atorvastatin dose-dependently decreases hepatic lipase activity in type 2 diabetes: effect of sex and the LIPC promoter variant. Diabetes Care 26:427-432

14. Baynes C, Henderson AD, Richmond W, Johnston DG, Elkeles RS (1992) The response of hepatic lipase and serum lipoproteins to acute hyperinsulinaemia in type 2 diabetes. Eur J Clin Invest 22:341-346

15. Jansen H, Verhoeven AJM, Weeks L et al (1997) Common C-to-T substitution at position -480 of the hepatic lipase promoter associated with a lowered lipase activity in coronary artery disease patients. Arterioscler Thromb Vasc Biol 17:2837-2842

16. Pihlajamäki J, Karjalainen L, Karhapää P et al (2000) G-250A substitution in promoter of hepatic lipase gene is associated with dyslipidemia and insulin resistance in healthy control subjects and in members of families with familial combined hyperlipidemia. Arterioscler Thromb Vasc Biol 20:1789-1795

17. Lewis GF, Murdoch S, Uffelman K et al (2004) Hepatic lipase mRNA, protein, and plasma enzyme activity is increased in the insulin-resistant, fructose-fed Syrian golden hamster and is partially normalized by the insulin sensitizer rosiglitazone. Diabetes 53:2893-2900

18. Carr MC, Hokanson JE, Deeb SS, Purnell JQ, Mitchell ES, Brunzell JD (1999) A hepatic lipase gene promoter polymorphism attenuates the increase in hepatic lipase activity with increasing intra-abdominal fat in women. Arterioscler Thromb Vasc Biol 19:2701-2707
19. Nimmo L, McColl AJ, Rosankiewicz JZ, Richmond W, Elkeles RS (1997) Regulation of hepatic lipase expression in HepG2 cells. Biochem Soc Trans 25:S689

20. Botma GJ, van Deursen D, Vieira D, van Hoek M, Jansen H, Verhoeven AJM (2005) Sterol-regulatory-element binding protein inhibits upstream stimulator factor-stimulated hepatic lipase gene expression. Atherosclerosis 179:61-67

21. Tu AY, Albers JJ (2001) Glucose regulates the transcription of human genes relevant to HDL metabolism. Diabetes 50:1851-1856

22. Botma GJ, Verhoeven AJM, Jansen H (2001) Hepatic lipase promoter activity is reduced by the C-480G and G-216A substitutions present in the common LIPC variant and is increased by upstream stimulatory factor. Atherosclerosis 154:625-632

23. Corre S, Galibert MD (2005) Upstream stimulating factors: highly versatile stress-responsive transcription factors. Pigment Cell Res 18:337-348

24. Iynedjian PB (1998) Identification of upstream stimulatory factor as transcriptional activator of the liver promoter of the glucokinase gene. Biochem J 333:705-712

25. Wang D, Sul HS (1997) Upstream stimulatory factor binding to the E-box at -65 is required for insulin regulation of the fatty acid synthase promoter. J Biol Chem 272:26367-26374

26. Nowak M, Helleboid-Chapman A, Jakel H et al (2005) Insulinmediated down-regulation of apolipoprotein A5 gene expression through the phosphatidylinositol 3-kinase pathway: role of upstream stimulatory factor. Mol Cell Biol 25:1537-1548

27. Ribeiro A, Pastier D, Kardassis D, Chambaz J, Cardot P (1999) Cooperative binding of upstream stimulatory factor and hepatic nuclear factor 4 drives the transcription of the human apolipoprotein A-II gene. J Biol Chem 274:1216-1255

28. Pastier D, Lacorte JM, Chambaz J, Cardot P, Ribeiro A (2002) Two initiator-like elements are required for the combined activation of the human apolipoprotein $C$-III promoter by upstream stimulatory factor and hepatic nuclear factor-4. J Biol Chem 277:15199-15206

29. Salero E, Gimenez C, Zafra F (2003) Identification of a non-canonical E-box motif as a regulatory element in the proximal promoter region of the apolipoprotein E gene. Biochem J 370:979-986

30. Vallet VS, Casado M, Henrion AA et al (1998) Differential roles of upstream stimulatory factors 1 and 2 in the transcriptional response of liver genes to glucose. J Biol Chem 273:20175-20179

31. Smih F, Rouet $P$, Lucas $S$ et al (2002) Transcriptional regulation of adipocyte hormone-sensitive lipase by glucose. Diabetes 51:293300

32. Bidder M, Shao JS, Charlton-Kachigian N, Loewy AP, Semenkovich CF, Towler DA (2002) Osteopontin transcription in aortic vascular smooth muscle cells is controlled by glucose-regulated upstream stimulatory factor and activator protein-1 activities. J Biol Chem 277:44485-44496

33. Weigert C, Brodbeck K, Sawadogo M, Häring HU, Schleicher ED (2004) Upstream stimulatory factor (USF) proteins induce human $T G F-b 1$ gene activation via the glucose-response element -1013 / -1002 in mesangial cells. J Biol Chem 279:15908-15915

34. Wang S, Skorczewiski J, Feng X, Mei L, Murphy-Ullrich JE (2004) Glucose up-regulates thrombospondin 1 gene transcription and transforming growth factor- $\beta$ activity through antagonism of cGMP-dependent protein kinase repression via upstream stimulatory factor 2. J Biol Chem 279:34311-34322

35. Ng MC, Miyake K, So WY et al (2005) The linkage and association of the gene encoding upstream stimulatory factor 1 with type 2 diabetes and metabolic syndrome in the Chinese population. Diabetologia 48:2018-2024

36. Pajukanta P, Lilja HE, Sinsheimer JS et al (2004) Familial combined hyperlipidemia is associated with upstream transcription factor 1 (USF1). Nat Genet 36:371-376

37. Coon H, Xin Y, Hopkins PN, Cawthon RM, Hasstedt SJ, Hunt SC (2005) Upstream stimulatory factor 1 associated with familial 
combined hyperlipidemia, LDL cholesterol, and triglycerides. Hum Genet 117:444-451

38. Komulainen K, Alanne M, Auro K et al (2006) Risk alleles of USF1 gene predict cardiovascular disease of women in two prospective studies. PloS Genetics 2:e69

39. Shoulders CC, Naoumova RP (2004) USF1 implicated in the aetiology of familial combined hyperlipidaemia and the metabolic syndrome. Trends Mol Med 10:362-365

40. Naukkarinen J, Gentile M, Soro-Paavonen A et al (2005) USF1 and dyslipidemias: converging evidence for a functional intronic variant. Hum Mol Genet 14:2595-2605

41. Putt W, Palmen J, Nicaud V et al (2004) Variation in USF1 shows haplotype effects, gene:gene and gene:environment associations with glucose and lipid parameters in the European Atherosclerosis Research Study II. Hum Mol Genet 13:15871597

42. Hoffstedt J, Ryden M, Wahrenberg H, van Harmelen V, Arner P (2005) Upstream transcription factor-1 gene polymorphism is associated with increased adipocyte lipolysis. J Clin Endocrinol Metab 90:5356-5360

43. van Deursen D, Botma GJ, Jansen H, Verhoeven AJM (2008) Down-regulation of hepatic lipase expression by elevation of cAMP in human hepatoma but not adrenocortical cells. Mol Cell Endocrinol doi: 10.1016/j.mce.2008.07.004
44. McMurray HR, McCance DJ (2003) Human papillomavirus type 16 E6 activates TERT gene transcription through induction of c-Myc and release of USF-mediated repression. J Virol 77:9852-9861

45. Van Linthout S, Riad A, Dhayat N et al (2007) Anti-inflammatory effects of atorvastatin improve left ventricular function in experimental diabetic cardiomyopathy. Diabetologia 50:1977-1986

46. Ma L, Robinson LN, Towle HC (2006) ChREBP*Mlx is the principal mediator of glucose-induced gene expression in the liver. J Biol Chem 281:28721-28730

47. Cartharius K, Frech K, Grote K et al (2005) MatInspector and beyond: promoter analysis based on transcription factor binding sites. Bioinformatics 21:2933-2942

48. Kawaguchi T, Osatomi K, Yamashita H, Kabashima T, Uyeda K (2002) Mechanism for fatty acid "sparing" effect on glucoseinduced transcription: regulation of carbohydrate-responsive element-binding protein by AMP-activated protein kinase. J Biol Chem 277:3829-3835

49. Jansen H, van Tol A, Auwerx J, Skretting G, Staels B (1992) Opposite regulation of hepatic lipase and lecithin: cholesterol acyltransferase by glucocorticoids in rats. Biochim Biophys Acta 1128:181-185

50. Jensen E, Hansson P, Floren CH, Nilsson A, Nilsson-Ehle P (1989) Cell-density-dependent release of hepatic lipase from cultured rat hepatocytes. Horm Metab Res 21:4-7 